\section{Adding chopped straw to concentrate feed: The effect of inclusion rate and particle length on intake behaviour of horses}

\author{
Andrea D. Ellis', Samantha Thomas', Kate Arkell² \\ and Patricia Harris ${ }^{3}$ \\ Nottingham Trent University, Southwell' ${ }^{1}$ SPILLERS ${ }^{\circledR}$, Milton Keynes ${ }^{2}$ and \\ WALTHAM Equine Studies Group, WALTHAM Centre for Pet Nutrition ${ }^{3}$
}

\section{Introduction}

Physiologically horses have evolved to consume structural herbage, which takes a considerable amount longer to chew than cereals or processed feeds. Previous research has highlighted a significant reduction in chewing rates for concentrate feed As the principal stimulus to saliva production in the horse is mastication, the amount of saliva produced therefore can also be compromised when feeding concentrates, which may influence subsequent digestion as well as buffering within the stomach. Dulphy et al. (1997) observed that the intake rates (dry matter per minute $=\mathrm{DM} / \mathrm{min}$ ) almost halved when comparing hay and straw $(14 \mathrm{~g}$ and $7.9 \mathrm{~g} \mathrm{DM} / \mathrm{min}$, respectively), highlighting the effect of physico-chemical composition on chewing efficiency in horses. for buffering stomach contents is also compromised. The inclusion of dried chopped forages or chaff has become popular but there are few published reports on the effects of chaff length or inclusion rate on concentrate feed intake behaviour. The purpose of this experiment study was to investigate the effects of increasing the amount of straw chaff to concentrate pelletswithin a meal on such behaviour of horses.

\section{Materials and Methods}

Feed intake behaviour (chews/minute, intake time $/ \mathrm{kg}$ Wet Matter [WM]) was measured in 6 mature horses (3 large horses: $670 \pm 19 \mathrm{~kg}$; 3 small horses: $508 \pm 12 \mathrm{~kg}$ ) morning and evening for the following feeds, fed in approximately $1 \mathrm{~kg}$ meals: their normal diet mix evening feed (NDM: pellets 50\%, Lucerne-straw chaff 15\%, and soaked sugarbeet pulp 35\%), this feed's normal diet pellets alone (NDP), a trial pellet (PP), a trial pellet-fibre mix (80\% pellets, 10\% molasses and 10\% short chopped lucerne) core pellet mixed with short chopped lucerne and (PF); PF the trial PF mix with added chopped straw (at two lengths - short $[\mathrm{S}] \sim 2.5 \mathrm{~cm}$; long $[\mathrm{L}] \sim 4 \mathrm{~cm}$ ) so that the added straw was present in the final mixture at three inclusion rates (PF10\%, 20\% and 30\% both for $L$ or S).

Both morning and evening (7.00 am and $17.00 \mathrm{pm}$ ) horses had not received any other feed for at least 3 hours prior to feeding times. Horses were offered $1-1.2 \mathrm{~kg}$ of each feed and feed was replaced after approximately $90 \%$ of feed had been eaten. Chews per minute were measured by direct observation technique and removed feed was weighed. Intake rate ( $\mathrm{g} /$ minute) and chewing rate (chews $/ \mathrm{kg}$ ) were calculated from measurements. Feed volume was measured according to $\mathrm{cm}^{3}$ occupied by a set level of each feed using volumetric flasks and a feed particle size index was calculated according to mean particle size of individual ingredients and by allowing for sieve mesh size used during pelleting procedure.

Trial feeds were fed according to a Latin Square design and the results analysed using analysis of variance after testing for normal distribution of data. The Spearman rank correlation test was used to test for associations. A p-value of less than 0.05 was taken as the lowest significance level.

\section{Results}

Mean intake behaviour results are given in Table 1. Multivariate analysis showed no effect of time of feeding on

Table 1 Mean intake behaviour of six horses ( \pm standard deviation).

\begin{tabular}{|c|c|c|c|c|c|}
\hline \multicolumn{3}{|r|}{ Chews/min ${ }^{b}$} & Chews/kg WM ${ }^{c}$ & $\begin{array}{l}\text { Intake time } / \mathrm{kg} \\
\text { WM (seconds) }\end{array}$ & $\begin{array}{l}\text { Intake rate } \\
(\mathrm{g} / \text { minute })^{c}\end{array}$ \\
\hline Feed $^{a}$ & $\mathrm{n}$ & Mean s.d. & Mean s.d. & Mean s.d. & Mean s.d. \\
\hline NDM & 23 & $77 \pm 12.8$ & $556 \pm 174.8$ & $429 \pm 108.8$ & $147 \pm 36.8$ \\
\hline & & $65 \pm 11.6$ & $472 \pm 113.8$ & $435 \pm 60.1$ & $140 \pm 19.4$ \\
\hline$P$ & 27 & $69 \pm 9.3$ & $446 \pm 99.0$ & $388 \pm 76.6$ & $161 \pm 32.1$ \\
\hline & $2 \varepsilon$ & $67 \pm 11.3$ & $609 \pm 185.7$ & $552 \pm 170.2$ & $113 \pm$ \\
\hline & 24 & $68 \pm 9.2$ & $735 \pm 149.8$ & $652 \pm 93.7$ & $94 \pm 15.7$ \\
\hline & 2 & $71 \pm 8.6$ & $735 \pm 120.1$ & $626 \pm 114.6$ & $99 \pm 19.4$ \\
\hline & 24 & $66 \pm 10.0$ & $896 \pm 189.8$ & $822 \pm$ & $75 \pm 13.1$ \\
\hline & 24 & $68 \pm 10.0$ & $918 \pm 205.4$ & $822 \pm 2$ & $77 \pm 15.6$ \\
\hline & 22 & $68 \pm 9.1$ & $1074 \pm 261.8$ & $950 \pm 183.5$ & $64 \pm 15.2$ \\
\hline$=30$ & 24 & $69 \pm 9.7$ & $1103 \pm 269.2$ & $954 \pm 201.4$ & $66 \pm 15$ \\
\hline
\end{tabular}

${ }^{a} \mathrm{NDM}=$ normal diet $\mathrm{mix}, \mathrm{NDP}=$ normal diet pellets, $\mathrm{PP}=$ trial mix pellets

$\mathrm{PF}=\mathrm{PP}$ and $10 \%$ chopped Lucerne, PF 10,20,30 = refers to $\%$ of chopped straw inclusion rate in $\mathrm{PF} ; \mathrm{L}=$ long straw $(\sim 4 \mathrm{~cm})$ and $\mathrm{S}=$ short straw $(\sim 2.5 \mathrm{~cm})$

measured

c calculated

chews/minute, chews $/ \mathrm{kg}$ WM or intake rate $\mathrm{g} / \mathrm{min}$. There was also no effect of horse group (according to size) on chews per minute. There was a significantly increased feed intake time and chews/kg WM for small horses $(p<0.01$, Figure 1).

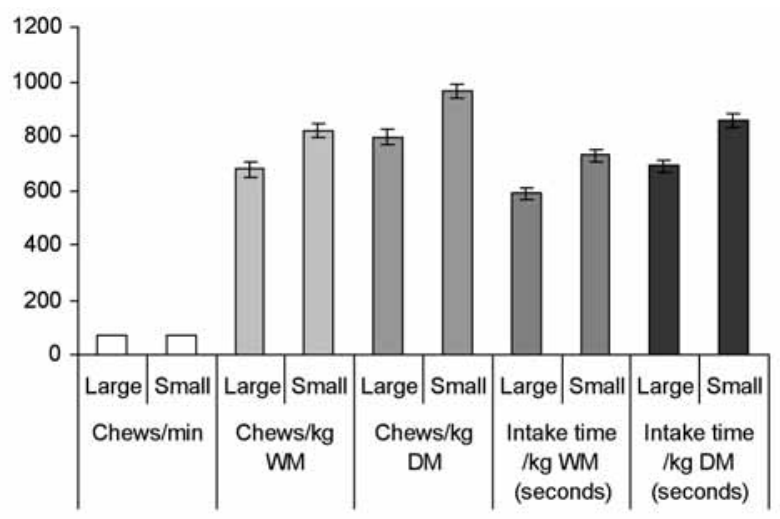

Fig 1 Mean feed intake behaviour for all feed according to horses size (Large or Small) (WM = wet matter; DM = dry matter).

There was a significant effect of type of feed on intake rates and chews/kg WM but no effect on chews/minute. Intake 
times ranged from 429 \pm 108 (NDM) to 954 \pm 201 (PF30L) seconds per $\mathrm{kg}$ feed as fed (WM). NDM, NDP, and PP were eaten much more quickly with fewer chews/kg WM than the diets with added straw $(p<0.001)$.

However, there was no significant difference between PF and PF10\% (609 \pm 185 vs $735 \pm 149$ chews/kg WM). In addition, there was no significant effect of adding more than $20 \%$ additional straw although the number of chews $/ \mathrm{kg}$ increased (20L $896 \pm 190$ vs 30 L $1074 \pm 262$ ).

There was no significant effect from straw chop length (see Figure 2). There was a significant correlation between feed volume and chews $/ \mathrm{kg}$ WM and between feed particle index and chews/kg WM (Spearman Rank Correlation, $p<0.01$; see Figure $3 . a \& b)$.

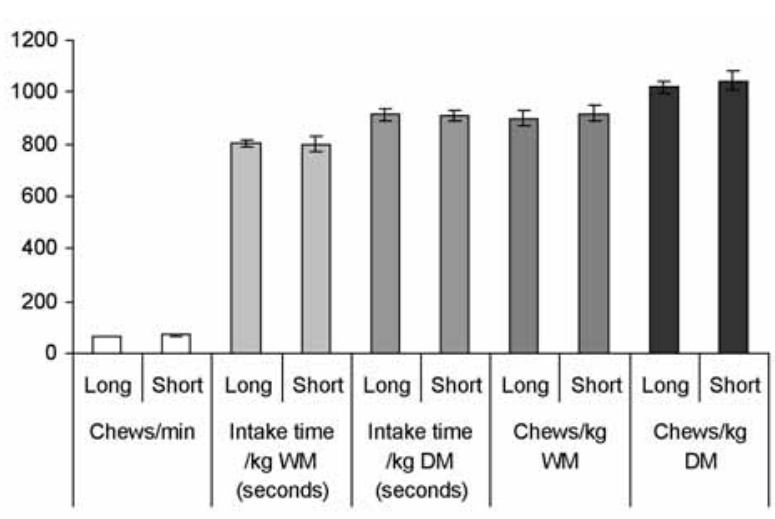

Fig 2 Mean Feed Intake behaviour (for PF10, PF20 and PF30, s.e.) according to straw chop length (Long $\sim 4 \mathrm{~cm}$ or Short $\sim 2.5$ $\mathrm{cm})$.

\section{Discussion}

Results show an increase in feed intake time and chews/minute relative to level of chaff inclusion for treatment feed (PF+). This indicates that adding chaff at an inclusion rate of $20 \%$ to a pellet-fibre mix will lead to significantly longer eating periods and increased chews $/ \mathrm{kg} W M(p<0.01)$. This is also likely to lead to an increased saliva production, thus increasing the buffering capacity against acid in the stomach. Meyer et al. (1986) reported that despite similar DM content of feed, hay boluses contain a much greater amount of wet matter $(827 \mathrm{~g} / \mathrm{kg}$ WM) than boluses retrieved from horses fed a mixed cereal feed $(685 \mathrm{~g} / \mathrm{kg}$ WM) and this effect was directly related to chewing time.

The correlation between feed volume and particle index further confirms these results with one exception. For the normal diet mix it is likely that the much greater wet matter content due to inclusion of soaked sugar beet has contributed to a faster intake rate and formation of bolus, although NDM consisted of a similar level of chaff inclusion and particle size index to the PF10S mix (see also NDM, Figure 3b). Interestingly due to the density and weight of sugarbeet NDM does not stand out in Figure 3a, where volume/weight ratio is measured. This is in accordance with previous observations (Ellis and Hill 2002). Therefore the beneficial effect of including chopped forages in concentrate feed may only be true for high dry matter feed. Further research in this area is necessary.
Horse size (3 Warmblood/lrish Draught type horses: $670 \pm$ $19 \mathrm{~kg}$; 3 small TB type horses: $508 \pm 12 \mathrm{~kg}$ ) in this study showed no effect on chews per minute, although previous studies report a direct relationship between occlusal surface area and chewing rates (Janis and Ehrhardt 1988, Meyer et al. 1975). The lower intake rates observed in the small horse group were therefore likely to be due to the smaller oral capacity of those animals (Meyer et al. 1975).

There was no effect on intake behaviour from particle length of chaff. It would therefore be recommended to investigate the effect of increased chaff length on passage rates and on faecal particle size, as efficiency of feed particle reduction has been directly linked to chewing behaviour in the past (Ellis and Hill 2002, Dulphy et al. 1997, Meyer et al. 1986).

a

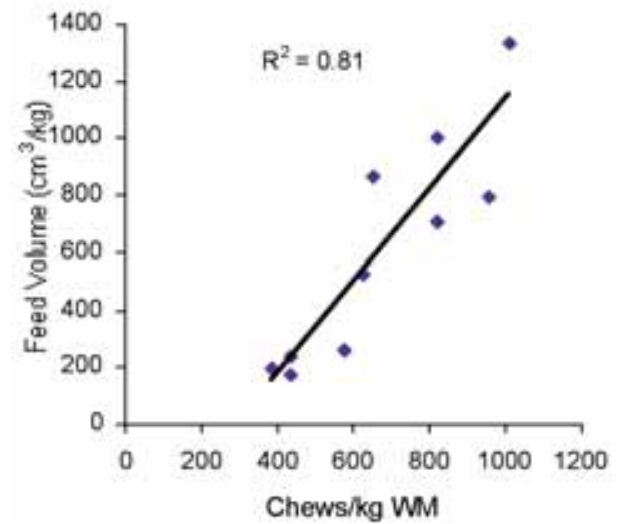

b

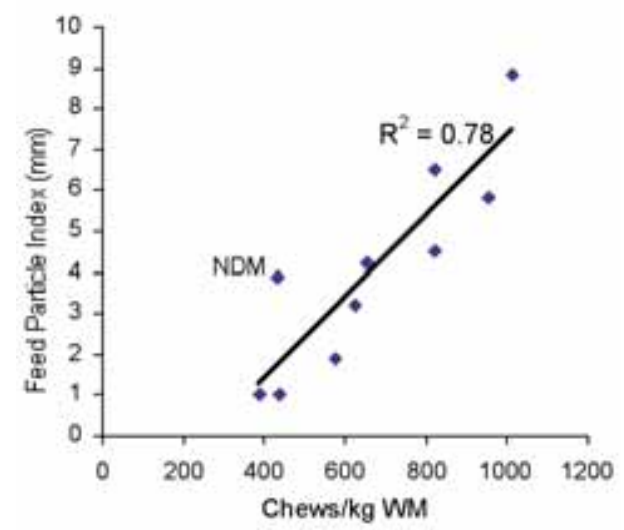

Fig 3 Correlation between chews/kg WM and Feed Volume (a) or Feed Particle Index (b) (NDM = normal diet mix).

\section{Conclusion}

A clear $120 \%$ increase in feed intake time and chews $/ \mathrm{kg}$ was observed as straw chaff was added to a high DM concentrate feed. Such an increase, at this level of intake, is likely to lead to a beneficial increase in saliva production without adversely affecting passage rate although further work is needed to confirm this.

\section{References}

Dulphy J. P., Martin-Rosset W., Dubroeuca H., Ballet J. M., Detour A. and Jailler M. (1997): Compared feeding patterns in ad libitum intake of dry forages by horses and sheep. Livestock Production Science 52, 49-56 
Ellis A. D. and Hill J. (2002): Feed factors affecting intake behaviour and water intake in horses. Proceedings of the 50th European Association of Animal Science, Cairo

Janis C. M. and Ehrhardt D. (1988): Correlation of relative muzzle width and relative insicor width with dietary preference in ungulates. Zoological Journal of the Linnean Society, 92, 267-284

Meyer H., Ahlswede L. and Reinhardt H. J. (1975): Studies on the duration of feeding, masticatory frequency and mincing of feed in horses. Deutsche Tierartztliche Wochenschrift, 82, 54-58
Meyer H., Coenen M. and Gürer C. (1986): Investigations on saliva production and chewing effects in horses fed various feeds. Proceedings of the 12th Equine Nutrition and Physiology Society, Gainesville, Florida, 92-97

\section{A. D. Ellis}

Nottingham Trent University

Southwell, NG25 OLZ

andrea.ellis@ntu.ac.uk

\section{The effect of the order of feeding oats and chopped alfalfa to horses on the rate of feed intake and chewing activity}

\author{
Nathalie Brüssow', Katrin Voigt', Ingrid Vervuert', Teresa \\ Hollands ${ }^{2}$, Derek Cuddeford ${ }^{3}$ and Manfred Coenen'
}

Institute of Animal Nutrition, University of Veterinary Medicine Hannover Foundation ${ }^{1}$, Dodson\&Horrell, Ringstead ${ }^{2}$ and Royal (Dick) School of Veterinary Studies, Roslin ${ }^{3}$ UK

\section{Introduction}

The speed of intake varies between $\sim 10 \mathrm{~min} / \mathrm{kg}$ for concentrates and $\sim 45 \mathrm{~min} / \mathrm{kg}$ for roughage. Associated with the rapid ingestion of concentrates per unit of time is the limited production of saliva $(<3 \mathrm{~kg} / \mathrm{kg}$ feed) whilst the consumption of roughage encourages a profuse production of saliva ( $\sim 5 \mathrm{~kg} / \mathrm{kg}$ feed, Meyer et al. 1975 1986). Food consumption accounts for the major part of the time budget of equids kept under natural conditions (Frentzen 1994). Concentrate feeding reduces the time spent feeding enabling other activities such as work. However, for the majority of horses this 'free' time is not used in useful activities. The consequent risk is that abnormal behaviours such as coprophagia or crib biting are performed and these present areas of concern for animal welfare (McGreevy et al. 1995; Waters et al. 2002). Another critical aspect of using concentrates is that of low saliva production. Obviously, there is no mechanical problem for the transport of the rather dry, ensalivated concentrate through the oesophagus, but problems may arise in the stomach (Vervuert and Coenen 2004) and may influence the function of the entire gut (Willard et al. 1977). Differences in saliva production per unit of feed affect the dry matter concentration of the gastric digesta. This interferes with digestive processes in the stomach, in particular, the acidification of the ingesta necessary to reduce microbial activity and to condition the material for digestion in the small intestine.
In order to offset the problems associated with the rapid consumption of concentrate it is common practice to recommend that chopped roughage is mixed with the concentrate or, that roughage be fed before providing the concentrate. However, the precise effects of these recommendations in terms of reducing the speed of intake are unknown. The objectives of the present study were to elucidate the effects of feeding oats alone before or after feeding chopped alfalfa or, in admixture with the alfalfa on feed intake and the chewing activity of healthy horses.

\section{Material and methods}

Four horses $(560 \pm 36 \mathrm{~kg} \mathrm{M})$ were used in a changeover experiment. The animals were individually kept on wood shavings in boxes with free access to water. The diets $(2$ meals/d) consisted of chopped alfalfa $(0.5 \mathrm{~g}$ crude fibre $=1.6 \mathrm{~g}$ alfalfa $/ \mathrm{kg}$ $\mathrm{Mx}$ meal- 1 ) and unprocessed oats (2 g starch $=4.5 \mathrm{~g}$ oats $/ \mathrm{kg}$ $M \times$ meal-1). The diets were offered alternatively in three ways; a) first alfalfa and immediately thereafter oats (A/O), b) first oats followed by alfalfa $(O / A)$ or $c)$, a mixture of alfalfa and oats (A-O-mix). Each diet was fed for 21 days. The time taken to consume the feed was recorded for each horse from 9 to up to 14 days depending on the type of feed. Parallel special halters were used which were equipped with a tube (fixed on the inner side of the strap which passes under the lower jaws), a pressure transducer and a data logger. Any jaw movement produced a signal via an increase in pressure in the tube (the indirect method). The signals were recorded by a Gemini Data Logger ${ }^{\circledR}$. Myography measurements were performed on two days for each horse per feeding session. Three electrodes were mounted superficially, one on the withers, the other two on the rising and the horizontal branch of the mandibles. The signals were amplified by an electronic amplifier (EMG Signalverstärker, IED ${ }^{\circledR}$ ). The software package DasyLab ${ }^{\circledR}$ was used as an interface for recording and reading of data. The data were analysed using an analysis of variance $\left(\right.$ Statistica $\left.{ }^{\circledR}\right)$ and the results are presented as mean \pm standard deviation (SD).

\section{Results}

The quickest intake was recorded with the alfalfa-oats mixture. The rate of intake of both oats and alfalfa was not influenced by the feeding order (Table 1). The chewing activity (chews/100 g) was similar for the A-O-Mix and oats at about 
Table 1 The mean $(\mathrm{n}=4)$ rate of intake $(\mathrm{g} / \mathrm{min})$ and chewing activity (chews $/ 100 \mathrm{~g}$ and chews $/ \mathrm{min}$ ) of horses fed oats or alfalfa separately and together 1) duration of one activity cycle 2) difference between min and max within a chewing cycle a. different superscript indicate significant differences, $p<0.05$.

\begin{tabular}{|c|c|c|c|c|}
\hline \multicolumn{5}{|c|}{ Indirect recording of jaw movement } \\
\hline \multicolumn{2}{|c|}{ diet } & $\mathrm{g} / \mathrm{min}$ & chews $/ 100 \mathrm{~g}$ & chews/min \\
\hline A-O-Mix & -- & $101 \pm 25.7^{\circ}$ & $83 \pm 15.3^{a}$ & $81 \pm 8.3$ \\
\hline \multirow[t]{2}{*}{$\mathrm{A} / \mathrm{O}$} & alfalfa lst & $64 \pm 10.2^{b c}$ & $132 \pm 27.8^{\text {bc }}$ & $82 \pm 8.1$ \\
\hline & oats 2 nd & $89 \pm 15.8^{\text {ad }}$ & $87 \pm 13.0^{\text {ad }}$ & $76 \pm 13.6$ \\
\hline \multirow[t]{2}{*}{$\mathrm{O} / \mathrm{A}$} & oats 1st & $104 \pm 16.8^{\text {ad }}$ & $81 \pm 7.9^{\text {ad }}$ & $84 \pm 13.0$ \\
\hline & alfalfa 2 nd & $59 \pm 13.7^{b c}$ & $147 \pm 25.2^{\text {bc }}$ & $80 \pm 12.1$ \\
\hline \multicolumn{5}{|c|}{ Myographical recording of jaw movement } \\
\hline \multicolumn{2}{|c|}{ diet } & chews/min & cycle, $\sec ^{1)}$ & amplitude $^{2 l}, \mathrm{mV}$ \\
\hline A-O-Mix & -- & $87 \pm 9.0$ & $0.21 \pm 0.019$ & $11.7 \pm 3.99$ \\
\hline \multirow{2}{*}{$\mathrm{A} / \mathrm{O}$} & alfalfa 1st & $82 \pm 10.6$ & $0.20 \pm 0.027$ & $8.9 \pm 3.33$ \\
\hline & oats 2 nd & $82 \pm 10.0$ & $0.21 \pm 0.027$ & $10.5 \pm 3.01$ \\
\hline \multirow[t]{2}{*}{$\mathrm{O} / \mathrm{A}$} & oats $1 \mathrm{st}$ & $81 \pm 9.7$ & $0.21 \pm 0.019$ & $10.0 \pm 3.85$ \\
\hline & alfalfa 2nd & $80 \pm 9.6$ & $0.21 \pm 0.043$ & $10.3 \pm 4.92$ \\
\hline
\end{tabular}

1) duration of one activity cycle ${ }^{2)}$ difference between min and max within

a chewing cycle

a.. different superscript indicate significant differences, $p<0.05$

90. More chewing was required during alfalfa ingestion independent of the order of feeding.

No differences exist between chews/min between the A-OMix or the roughage and oats. The indirect method and myography yielded comparable results for chewing activity. The mean duration of a chewing cycle was $0.2 \mathrm{sec}$ with no influence of feeds or feeding order; there was a trend towards shorter cycles towards the end of a meal (Figure 1). Further time-associated changes were, an increase in the interval between chewing episodes and a reduction in the amplitude. Chews/min were unaffected by type of feed and feeding order.

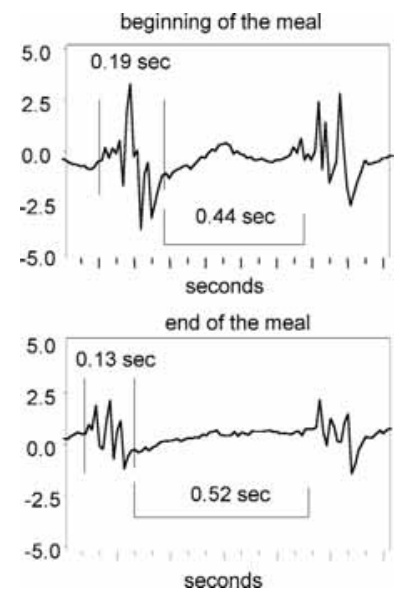

Fig 1 Examples of two chewing cycles measured by myography in one horse at the beginning and end of a meal (A-OMix); one chewing cycle is defined as the 0.13-0.19 s lasting sequence of spikes.

\section{Discussion}

There is a close interaction between nutrition and behaviour. Negative effects may be seen amongst stabled horses (Vervuert and Coenen 2001). Stereotypic behaviour is a common problem in horses that are isolated and inactive. Therefore, any feeding regime that could increase the time spent feed intake would be beneficial. The hypothesis that, by simply mixing roughage with concentrate the rate of food consump- tion will be reduced, is flawed. About $60 \mathrm{~g}$ alfalfa are consumed per min whilst oats are ingested at 90-100 g per min; mixing both components together seems to speed up intake. Since both feeds are highly palatable any confounding effects of feed preference can be ignored. A lower rate of intake was expected when forage was fed first but this was not confirmed by the results of the current study. The order in which the feeds were supplied was insufficient to influence the rate of intake (g/min). The chewing frequency (chews/min) showed little variation between the diets and in the order of feeding. As saliva production is associated with chewing activity, it can be concluded that saliva production per min was comparable. Consequently the chews/g of feed determines salivation during ingestion and alfalfa stimulates more chewing and thus more salivation than oats.

\section{Conclusion}

Horses have fairly uniform chewing frequencies of 80-85 chews/min. Variations in feed intake are thus not related to changes in chewing frequency but rather to the need to reduce particle size. Neither feeding oats after forage nor by mixing them with forage reduced the speed of intake or the number of chews per unit of feed increased. The ratio of roughage to oats used in the current study $(\sim 1: 3)$ is different from the ratio in common rations. Thus, further experiments are required to discover more about fibre/concentrate interactions. Cereal/fibre mixes of the type used in the current study appear to be of little benefit in ameliorating the rapidity with which concentrates are consumed.

\section{References}

Frentzen F. (1994): Bewegungsaktivitäten und Verhalten von Pferden in Abhängigkeit von Aufstallungsform und Fütterungsrhythmus unter besonderer Berücksichtigung unterschiedlich gestalteter Auslaufsysteme. Vet. Med. Diss. Hannover

McGreevy P. D., Cripps P. J., French N. P., Lee L. E. and Nicol C. J. (1995): Management factors associated with stereotypic and redirected behaviour in the Thoroughbred horse. Equine Vet J 27, 86-91

Meyer H., Ahlswede L. and Reinhard H. (1975): Untersuchungen über Freßdaver, Kaufrequenz und Futterzerkleinerung beim Pferd. Dtsch. Tierärztl Wschr 82, 54-58

Meyer H., Coenen M. and Probst D. (1986): Beiträge zur Verdauungsphysiologie des Pferdes. 14. Mitteilung: Futtereinspeichelung und -passage im Kopfdarm des Pferdes. J Anim Physiol a Anim Nutr 56, 171-183

Vervuert I. and Coenen M. (2001): Aspekte der Fütterungs- und Haltungstechnik von Pferden. Übers Tierernährg 29, 131-138

Vervuert I. and Coenen M. (2004): Nutritive Risiken für das Auftreten von Magengeschwüren beim Pferd. Pferdeheilkunde 20, 349-352

Waters A. J., Nicol C. J. and French N. P. (2002): Factors influencing the development of stereotypic and redirected behaviours in young horses: findings of a four year prospective epidemiological study. Equine Vet J 34, 572-579

Willard J. G., Wolfram S. A. and Baker J. P. (1977): Effect of diet on cecal $\mathrm{pH}$ and feeding behavior of horses. J Anim Sci 45, 87-93

\section{Coenen}

Institute of Animal Nutrition

University of Veterinary Medicine Hannover - Foundation

Bischofsholer Damm 15

30173 Hannover,

manfred.coenen@tiho-hannover.de 